\title{
Astaxanthin protects spinal cord tissues from apoptosis after spinal cord injury in rats
}

\author{
Shuang Li1" ${ }^{1 \#}$ Xiaofan Gao ${ }^{2 \#}$, Qiushuang Zhang ${ }^{3}$, Xin Zhang ${ }^{4}$, Wei Lin ${ }^{2}$, Wei Ding ${ }^{2}$ \\ ${ }^{1}$ Department of Ear-Nose-Throat Surgery, The Second Hospital of Jilin University, Changchun, China; ${ }^{2}$ Department of Thyroid Surgery, The \\ Second Hospital of Jilin University, Changchun, China; ${ }^{3}$ Department of Urology, The Second Hospital of Jilin University, Changchun, China; \\ ${ }^{4}$ Department of Cataract, The Second Hospital of Jilin University, Changchun, China \\ Contributions: (I) Conception and design: S Li, X Gao, W Lin, W Ding; (II) Administrative support: W Lin, W Ding; (III) Provision of study \\ materials or patients: W Lin, W Ding; (IV) Collection and assembly of data: S Li, X Gao, Q Zhang; (V) Data analysis and interpretation: S Li, X \\ Gao, X Zhang; (VI) Manuscript writing: All authors; (VII) Final approval of manuscript: All authors. \\ \#These authors contributed equally to this work. \\ Correspondence to: Wei Lin; Prof. Wei Ding, MD, PhD. Department of Thyroid Surgery, The Second Hospital of Jilin University, Changchun \\ 130041, China. Email: 2570051137@qq.com; 1095732328@qq.com.
}

Background: There is emerging evidence that astaxanthin plays a significant role in protecting neuro-cells from apoptosis after central nervous system injury. Our study examined the effects of astaxanthin on neurocell apoptosis after spinal cord injury.

Methods: One hundred and forty-four healthy adult Sprague-Dawley rats were randomly divided into the experimental group, control group, and sham operation group $(n=48)$. Spinal cord injury was induced using the modified Allen method in the control group and the experimental group; while in the sham operation group, the lamina was removed without spinal cord injury. The rats in the experimental group were given astaxanthin $(75 \mathrm{mg} / \mathrm{kg}$ ) by gavage immediately after the operation, and in the other groups were given the same amount of olive oil. Motor function was assessed by blood-brain barrier (BBB) scores. The malondialdehyde (MDA) content and the activity of superoxide dismutase (SOD) at 24 h was determined after the operation. The apoptosis index (AI) was determined at 6, 24, and $48 \mathrm{~h}$ after the operation. At $48 \mathrm{~h}$ after the operation, we calculated the water content of the spinal cord, the lesion ratio of the spinal cord, the ultrastructure of the spinal cord, and the ultrastructure score.

Results: The BBB scores of the control and experimental groups were significantly lower than the sham operation group at each postoperative time $(\mathrm{P}<0.05)$, and the $\mathrm{BBB}$ score of the experimental group was significantly higher than the control group at $1-4$ weeks postoperatively $(\mathrm{P}<0.05)$. At 24 hours postoperatively, MDA content was highest in the control group and lowest in the sham operation group, while SOD activity was highest in the sham operation group and lowest in the control group $(\mathrm{P}<0.05)$. At each time point postoperatively, the sham operation group had the lowest AI and the control group had the highest $\mathrm{AI}(\mathrm{P}<0.05)$. At $48 \mathrm{~h}$ after the operation, the water content and the lesion ratio of the spinal cord, and the ultrastructure score were the lowest in the sham operation group and the highest in the control group $(\mathrm{P}<0.05)$.

Conclusions: Astaxanthin significantly improved the motor function of rats with spinal cord injury.

Keywords: Astaxanthin; spinal cord injury; apoptosis; rat

Submitted Oct 22, 2021. Accepted for publication Dec 23, 2021.

doi: 10.21037/atm-21-6356

View this article at: https://dx.doi.org/10.21037/atm-21-6356 


\section{Introduction}

Astaxanthin, which is a carotenoid widely found in the biological world, has a wide range of biological effects, including anti-inflammatory, antioxidant, and anti-tumor, and anti-aging effects, prevents cerebrovascular diseases, and regulates the immune system (1-4). There is emerging evidence that astaxanthin plays a significant role in protecting neuro-cells from apoptosis after central nervous system injury, such as brain trauma, cerebral ischemic injury, cerebral ischemia reperfusion injury, Parkinson's syndrome, and Alzheimer's syndrome (1-5). However, its effects on spinal cord injury are unclear. Thus, this study sought to examine the effects of astaxanthin on neuro-cell apoptosis after spinal marrow injury in rats, and observe ultrastructure and motor function changes.

We present the following article in accordance with the ARRIVE reporting checklist (available at https://dx.doi. org/10.21037/atm-21-6356).

\section{Methods}

\section{Animals and regents}

One hundred and forty-four clean, healthy rats (male and female), weighing $280 \pm 20 \mathrm{~g}$, were provided by the Animal Experimental Center of Jilin University. Astaxanthin (Sigma, USA) was dissolved in olive oil immediately before use. We also obtained a malondialdehyde (MDA) detection kit, superoxide dismutase (SOD) detection kit (Nanjing Jiancheng Institute of Biological Engineering, Nanjing, China), apoptosis detection kit (Wuhan Boster Bioengineering Technology., Ltd., Wuhan, China), Motic Med 6.0 Digital Medical Image Analysis System [Motic (Xiamen) Medical Diagnostic Systems Co., Ltd., Xiamen, China], JEM-1200EX transmission electron microscope (Japan), and LKB-5 ultra-thin microtome (LKB company, Sweden). A protocol was prepared before the study without registration. Experiments were performed under a project license granted by Institutional Animal Care and Use Committee of Jilin University, in compliance with Institutional Animal Care and Use Committee of Jilin University guidelines for the care and use of animals.

\section{Groups and methods}

One hundred and forty-four Sprague-Dawley rats were equally allocated to the: (I) control group; (II) sham operation group; and (III) experimental group using the random-number table method. The rats were anesthetized and intraperitoneally injected with $1 \%$ sodium pentobarbital $(40 \mathrm{mg} / \mathrm{kg})$, and then placed in a prone position on a table. After anesthesia, the T10 spinous was taken as the center maker, a 5 -cm incision was made on the posterior midline, and the sciatic nerve was crosscut under the greater trochanter of the femur $0.5-1 \mathrm{~cm}$ from the inferior margin of the piriform muscle. The skin and subcutaneous tissue were cut in turn, and peeled off the paravertebral muscles to expose the spines protuberance and lamina. The T10 spinous process and total lamina were removed, and the $\mathrm{T} 9$ and $\mathrm{T} 10$ lamina were partially removed to enlarge the spinal canal. The sham operation group only received incision sutures. In the control group and the experimental group, the 20-mm spinal cord was exposed, and the model of the spinal cord injury was made using a modified Allen method. Spinal cord injury was induced by a self-made modified Allen's stall with damage energy of $50 \mathrm{~g}-\mathrm{cm}$ force $(5 \mathrm{~g} \times 10 \mathrm{~cm})$. The model was considered to have been successfully established if the following occurred: hemorrhage and edema during the spinal marrow injury, spastic swinging of the tail, fluttering and the retraction of both the lower extremities and body, and lower extremity paralysis. After the model was successfully established, the incision was sutured layer by layer. The rats in the experimental group were intragastrically administered astaxanthin $[75 \mathrm{mg} / \mathrm{kg}(6)]$ twice per day after the operation, while the rats in other groups were administered equal amounts of olive oil twice per day. The rats had free access to food and drink, and were housed in a room with a temperature of $25-27^{\circ} \mathrm{C}$ on a $12 \mathrm{~h}$ day/night cycle. Artificial bladder urination was performed 3 times per day, until a reflex bladder formed.

\section{Statistical analysis}

SPSS 20.0 software was used for the statistical analysis. The measurement data are showed as mean \pm standard deviation. A single-factor analysis of variance was used to compare each group, and the least significant difference test was adopted for the pairwise comparison. $\mathrm{P}<0.05$ was considered to be statistically significant.

\section{Observation indexes}

\section{Motor function evaluation}

Blood-brain barrier (BBB) scores were used to assess the motor function of the six rats in each group at day 1 , and $1,2,3$, and 4 weeks post-operation. Double-blind scoring 
was performed by two researchers familiar with the scoring standards.

\section{Determination of the MDA content and SOD activity}

Rats in each group were sacrificed $24 \mathrm{~h}$ after the operation, and their spinal cord tissues were taken under anesthesia. Specifically, $10 \mathrm{~mm}$ of spinal cord tissue was removed from the injury point, and then weighed and homogenized. MDA content was determined using the thiobarbituric acid method in accordance with the manufacturer's instructions. The xanthine oxidase method was used to determine SOD activity in accordance with the method described in the SOD detection kit.

\section{Cell apoptosis}

Six rats from each group were sacrificed at 6, 24, and $48 \mathrm{~h}$ after the operation. A midline incision was made through the skin along the thorax. Heart intubation and perfusion fixation were then performed after anesthesia. Ten $\mathrm{mm}$ of spinal cord tissue was removed from the injury point, trimmed and embedded in paraffin (continuous slices were made; slice thickness: $5 \mu \mathrm{m}$ ). The apoptosis detection kit was used strictly according to the instructions, and the slices were observed under a light microscope. The apoptotic cells were labeled as brown-yellow using the in-situ endlabeling method. A high-power field was randomly selected from each slice to count the apoptosis index (AI) using the following formula: (apoptotic cells/total cells) $\times 100 \%$. The results were independently observed by two pathologists in accordance with the double-blind principle.

\section{Evaluation of spinal cord tissue edema}

The dry-wet weight method was used to determine the water content of the spinal cord tissue, and evaluate the degree of spinal marrow tissue edema. 6 rats from each group were sacrificed $48 \mathrm{~h}$ after the operation, and $10 \mathrm{~mm}$ of spinal cord tissue was removed from the injury point under hypothermia. The spinal cord tissues were weighed immediately after preparation (wet weight, $W$ ) and after drying in an oven at $80{ }^{\circ} \mathrm{C}$ for $48 \mathrm{~h}$ (dry weight, D); this was repeated 3 times and the average was taken. The water content of the spinal cord tissue was calculated using the following formula: $[(W-D) / W] \times 100 \%$. The score indicated the correlation between the value and degree of spinal cord edema.

\section{Spinal cord injury area determination}

Six rats from each group were sacrificed $48 \mathrm{~h}$ after the operation, and spinal cord tissue was removed from the injury point. The specimens were processed according to the protocol of light microscope specimen preparation. The slices, which had a thickness of $25 \mu \mathrm{m}$, were routinely stained with hematoxylin and eosin. The Motic Med 6.0 digital medical image analysis system was used to measure the pixels of the injury and the total pixels of the cross-section of the spinal cord. The calculated ratio of the 2 indicated the area ratio of the spinal cord injury.

\section{Ultrastructure observation and scoring}

Six rats from each group were sacrificed at $48 \mathrm{~h}$ after the operation. The specimens were processed in steps according to the requirements of transmission electron microscope specimen preparation, sliced with an LKB-5 ultra-thin microtome to a thickness of 50-60 nm, stained with lead citrate, and observed with a JEM-1200EX transmission electron microscope. The specimens were examined under $\times 5,000$ magnification, and the Kaptanoglu scoring method was used (7). The ultrastructural scoring of the specimens was performed using the transmission electron microscope, and the ultrastructural changes of 20 nerve cells, 20 axons, and 20 mitochondria were evaluated for each specimen. Intracytoplasmic edema, nuclear damage, axon degeneration, myelin breakdown, and mitochondrial damage scores were calculated for each group. The values were positively correlated with the spinal cord injury severity. Two researchers performed the double-blind scoring calculations.

\section{Results}

\section{Motor function evaluation}

At each time point after surgery, the BBB scores were significantly lower in the control and experimental groups than the sham operation group $(\mathrm{P}<0.05)$. At $1-4$ weeks after surgery, the $\mathrm{BBB}$ scores of the experimental group were significantly higher than those of the control group $(\mathrm{P}<0.05$; see Table 1).

\section{Determination of MDA content and SOD activity}

At $24 \mathrm{~h}$ after surgery, the MDA content of the spinal cord in the control group, experimental group, and sham operation group were $2.82 \pm 0.24,10.12 \pm 0.64$, and $6.21 \pm 0.36 \mathrm{nmol} / \mathrm{mg}$, respectively. Thus, the MDA content of the control group and the experimental group was 
Table 1 Comparison of BBB scores of rats in each group at each time point after the operation $(\mathrm{n}=6, \bar{x} \pm s)$

\begin{tabular}{lccccc}
\hline Group & $1 \mathrm{~d}$ & 1 week & 2 weeks & 3 weeks & 4 weeks \\
\hline Sham & $18.8 \pm 0.4^{\#}$ & $21.0 \pm 0.0^{\#}$ & $21.0 \pm 0.0^{\#}$ & $21.0 \pm 0.0^{\#}$ & $21.0 \pm 0.0^{\#}$ \\
Control & $0^{*}$ & $2.3 \pm 0.4^{*}$ & $3.8 \pm 0.5^{\star}$ & $6.0 \pm 0.3^{*}$ & $8.2 \pm 0.6^{\star}$ \\
Experimental & $0^{*}$ & $4.2 \pm 0.6^{* \#}$ & $6.6 \pm 0.8^{\star \#}$ & $9.8 \pm 0.5^{* \#}$ & $12.6 \pm 0.4^{\star \#}$ \\
Statistic & $\mathrm{F}=13,254.000 ; \mathrm{P}=0.000$ & $\mathrm{~F}=3,666.577 ; \mathrm{P}=0.000$ & $\mathrm{~F}=1,722.607 ; \mathrm{P}=0.000$ & $\mathrm{~F}=3,219.529 ; \mathrm{P}=0.000 \quad \mathrm{~F}=1,464.000 ; \mathrm{P}=0.000$ \\
\hline
\end{tabular}

*, compared to the sham group, $\mathrm{P}<0.05$; ", compared to the control group, $\mathrm{P}<0.05$. BBB, blood-brain barrier.
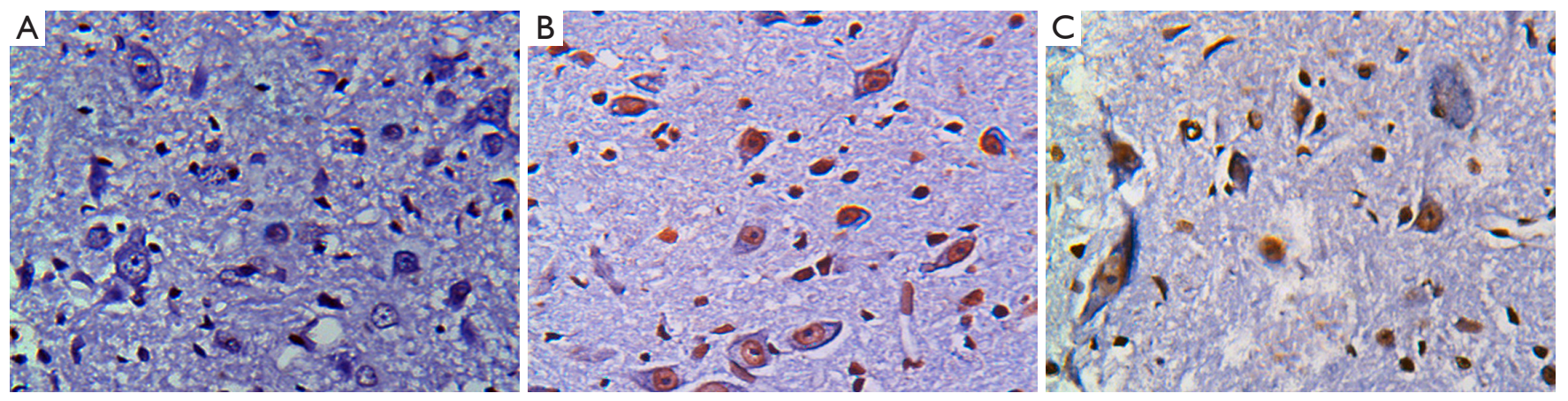

Figure 1 TUNEL staining in each group at $48 \mathrm{~h}$ after the operation $(\times 200)(\mathrm{A})$ Sham group; (B) control group; (C) experimental group.

Table 2 Comparison of AIs of rats in each group at each time point after the operation $(\mathrm{n}=6, \%, \bar{x} \pm s)$

\begin{tabular}{lccc}
\hline Group & $6 \mathrm{~h}$ & $24 \mathrm{~h}$ & $48 \mathrm{~h}$ \\
\hline Sham & $0^{\#}$ & $0^{\#}$ & $0^{\#}$ \\
Control & $13.8 \pm 1.6^{\star}$ & $31.2 \pm 2.4^{*}$ & $34.2 \pm 2.8^{\star}$ \\
Experimental & $10.4 \pm 1.2^{\star \#}$ & $14.2 \pm 1.6^{\star \#}$ & $18.4 \pm 2.1^{\star \#}$ \\
Statistic & $\mathrm{F}=232.620 ;$ & $\mathrm{F}=527.913 ;$ & $\mathrm{F}=430.491 ;$ \\
& $\mathrm{P}=0.000$ & $\mathrm{P}=0.000$ & $\mathrm{P}=0.000$ \\
\hline
\end{tabular}

*, compared to the sham group, $\mathrm{P}<0.05$; ", compared to the control group, $\mathrm{P}<0.05$. Al, apoptosis index.

significantly higher than that of the sham operation group, and the MDA content of the experimental group was significantly lower than that of the control group $(\mathrm{P}<0.05)$. The SOD activity results of the spinal cord tissues from the control group, experimental group, and sham operation group were $35.63 \pm 1.76,17.83 \pm 1.21$, and $31.56 \pm 1.46 \mathrm{U} / \mathrm{mg}$ respectively. Thus, the SOD activity of the control group and the experimental group was significantly lower than that of the sham operation group, and that of the experimental group was higher than that of the control group $(\mathrm{P}<0.05)$.

\section{Apoptosis detection}

There were no TUNEL positive cells in the sham operation group at any time point after surgery. More TUNEL positive cells of different sizes and uneven distribution in both gray matter and white matter were found in the control group than the experimental group (see Figure 1). At each time point after surgery, the AIs were significantly higher in the control and experimental groups than the sham operation group, and significantly lower in the experimental group than the control group $(\mathrm{P}<0.05$; see Table 2).

\section{Evaluation of spinal cord tissue edema}

Forty-eight hours after the operation, the spinal cord water content of the sham operation group, control group, and experimental group were $55.21 \% \pm 1.89 \%, 88.11 \% \pm 4.12 \%$, and $64.84 \% \pm 3.06 \%$, respectively. Thus, the control group and experimental groups had significantly higher spinal cord water content than the sham operation group, and the experimental group had significantly lower spinal cord water content than the control group $(\mathrm{P}<0.05)$. 

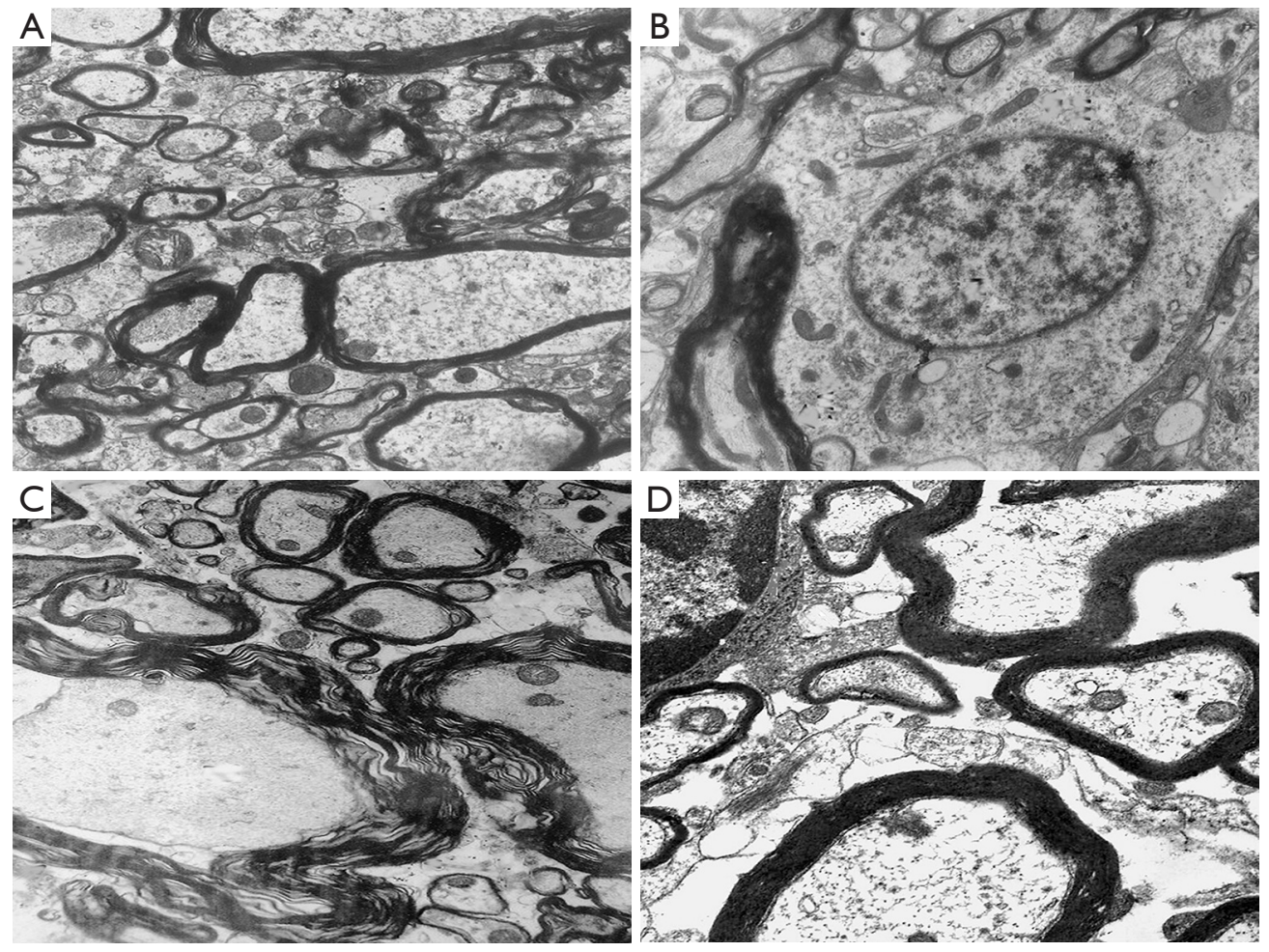

Figure 2 Transmission electron microscope observation at $48 \mathrm{~h}$ after the operation $(\times 5,000)$. (A,B) The gray matter and white matter of the spinal cord in the sham group; (C) the white matter of the spinal cord in the control group; (D) the white matter of the spinal cord in the experimental group.

\section{Spinal cord injury area determination}

Forty-eight hours after the operation, the spinal cord injury area ratios of the sham operation group, control group, and experimental group were $0,0.52 \pm 0.08$, and $0.28 \pm 0.05$, respectively. Thus, the spinal cord injury area ratios of the control group and the experimental group were significantly higher than that of the sham operation group, while the spinal cord injury area ratio of the experimental group was significantly lower than that of the control group $(\mathrm{P}<0.05)$.

\section{Ultrastructure observation and score}

Transmission electron microscopy showed no hemorrhages or edemas in the spinal marrow of rats in the sham operation group $48 \mathrm{~h}$ after the operation. Indeed, the normal spinal cord tissues, nerve cells, glial cells, nerve fibers, membranous organelles, axons, and myelin of the rats in the sham operation group all had normal ultrastructure. Hemorrhages and edemas were observed in the spinal cord tissue of rats in the control group. Significant degeneration of the nucleus and cytoplasmic organelles of the nerve cells in the gray matter, myelin division and changes in the axon organelles of myelinated nerve fibers in the white matter, edema around capillaries, and organelle changes of capillary endothelial cells were also observed in the control group. Interstitial edemas and hemorrhages were observed in some areas of the spinal cord tissue in the experimental group, but this was less severe than that of the control group. Some nerve cell membrane organelles in the gray matter were vacuolated, lipofuscin granules were aggregated, the structure of the glial cells was basically normal, the myelin sheath of the myelinated nerve fibers in the white matter was partially separated, most of the nerve fibers showed normal ultrastructure, and some capillaries showed edema but their structure was normal (see Figure 2). The ultrastructure scores of the sham operation group, control group and experimental group were $0,8.68 \pm 0.32$, and $3.12 \pm 0.16$, respectively. The ultrastructural scores of the control group and the experimental group were significantly higher than that of the sham operation group, and the ultrastructural score of the experimental group was 


\section{Page 6 of 8}

significantly lower than that of the control group $(\mathrm{P}<0.05)$.

\section{Discussion}

Spinal cord injury generally includes primary injury and secondary injury. Primary injury refers to the destruction of nerve cells, axonal rupture, cytoplasmic membrane rupture, and endolysin release, which lead to cell death after spinal cord damage caused by violence. A sequential tissue self-destruction process involving multiple factors that occur on the basis of primary injury is called secondary injury. Apoptosis, also known as programmed cell death, is an inherent natural physiological process that maintains the stability of systems and tissues by removing dead cells and metabolic products. Some research has shown that apoptosis, which results in the death of neurons and glial cells, plays an important role in secondary spinal cord injury in humans and animals. Li et al. (8) first identified apoptosis after spinal cord injury using the in-situ end-labeling method (the TUNEL method) in a compressive spinal cord injury model. Numerous positive cells in the white matter of the cranial and caudal spinal marrow were found at $4 \mathrm{~h}$ after injury, and the highest numbers of positive cells were observed from the 1st to 3 rd week after injury. Yong et al. (9) confirmed apoptosis induced by severe spinal cord injury in rats, and observed that the apoptotic cells are mainly neurons, astrocytes, oligodendrocytes, and microglia. Researchers have continuously observed spinal cord contusion models in rats at different periods from 5 minutes to 30 days, and found that the central injury area appeared at the 5 th minute after spinal cord injury without apoptosis, and apoptosis in the injured area appeared in neurons after $4 \mathrm{~h}$, and reached its peak at $8 \mathrm{~h}$ (10). Apoptosis in glial cells appeared at $4 \mathrm{~h}$ after injury and reached its peak at $24 \mathrm{~h}$. Within 1 week of the injury, the initial injury area gradually expanded and cavities appeared, while more neurons and glial cells died successively. Liu et al. (10) contended that this pathological process may be related to demyelination changes in the remaining axons. Crowe et al. (11) suggested that apoptosis coexists with necrosis after the destruction of the central nervous system, which leads to damage of the central nervous system. Our experiment showed that the AI of the spinal cord tissue of the control group was significantly higher than that of the sham operation group at any point in time $(\mathrm{P}<0.05)$, indicating that severe apoptosis occurred after spinal cord injury. This study also found that the content of MDA in the spinal cord of the control group was significantly higher than that of the sham operation group

\section{Li et al. Astaxanthin protects the spinal cord tissues from injury}

$(\mathrm{P}<0.05)$, and the SOD activity was significantly lower than that of the sham operation group $(\mathrm{P}<0.05)$, implying that spinal cord injury decreases the ability of antioxidant enzymes, which induces the excessive oxygenation of free radicals and severe lipid peroxidation. The water content of spinal marrow, the damage ratio of the spinal marrow, and the ultra-microstructure scores in the control group were significantly higher than those in the sham operation group $(\mathrm{P}<0.05)$. The BBB scores in the control group and the experimental group were evidently lower than that in the sham operation group at each postoperative time point $(\mathrm{P}<0.05)$; and the $\mathrm{BBB}$ score in the experimental group were evidently higher than in the control group at $1-4$ weeks postoperatively $(\mathrm{P}<0.05)$. Thus, the spinal cord injury induced spinal cord edema, spinal cord histopathological damage, ultrastructural damage, and motor dysfunction in rats.

Astaxanthin, also known as lobster shell pigment, is a kind of deep pink carotenoid, is similar to $\beta$-carotene in chemical structure, and is also the highest grade product of carotenoid synthesis. Studies have shown that astaxanthin is a powerful antioxidant with anti-inflammatory, antioxidant, anti-tumor, and other pharmacological effects $(1,2)$. There is emerging evidence that astaxanthin effectively protects cells from apoptosis after brain injury, maintains neurofunction, and improves neuroprotective effects (12). In an experimental subarachnoid hemorrhage model, $\mathrm{Wu}$ et al. (5) found that by activating nuclear factor related factor 2 and the antioxidant response factor pathways can reduced oxidative damage, brain edema, and damage to the BBB, inhibited cell apoptosis, and improved neurofunction and brain injury. In a rat subarachnoid hemorrhage model, astaxanthin was found to significantly increase the level of phosphorylated Akt and Bad in the cerebral cortex, while significantly reducing the level of caspase-3, which demonstrates its protective effects on the brain after subarachnoid hemorrhage in rats (13). Wang et al. (13) confirmed that in a isoflurane-induced brain injury model, astaxanthin deactivated caspase-3, protected cells from apoptosis, promoted cell growth, and inhibited isoflurane-induced neuronal apoptosis by upregulating the phosphatidylinositol 3 kinase/protein kinase B pathway. Additionally, astaxanthin also plays an important role in neuroprotection by inhibiting NF- $\mathrm{KB}$ nuclear translocation and the activity of caspase-3, decreasing the expression of pro-inflammatory cytokines, sodium, potassium, and chloride co-transporters, and decreasing the level of Bax while increasing the level of Bcl-2, which helps to reduce 
the apoptosis of astrocytes (14). Pan (15) also showed that astaxanthin enhanced the antioxidant capacity, activated the antioxidant defense pathway, inhibited reactive oxygen species and apoptosis, improved nerve regeneration, reduced cerebral infarction volume and improved neurofunction in a rat cerebral ischemia model. Our results confirm that astaxanthin not only inhibits apoptosis after spinal cord injury (see Table 2), but also enhances the activity of antioxidant enzymes, which reduce excessive oxygenation of free radicals and lipid peroxidation after spinal cord injury. Additionally, astaxanthin significantly reduces spinal cord edema, spinal histopathological and ultrastructural damage, and protects spinal cord tissue and motor function in rats with spinal cord injury.

In short, our findings provide a potential clinical treatment for spinal cord injury; however, the experimental group and the sham operation group showed significant differences; thus, the neuroprotective effects of astaxanthin on spinal cord injury require further research.

\section{Acknowledgments}

Funding: This work was supported by "The Fifteen" Science and Technology Research Program of Jilin Provincial Department of Education (JJKH20201086KJ) and the Jilin University Horizontal Subject (2019YX608).

\section{Footnote}

Reporting Checklist: The authors have completed the ARRIVE reporting checklist. Available at https://dx.doi. org/10.21037/atm-21-6356

Data Sharing Statement: Available at https://dx.doi. org/10.21037/atm-21-6356

Conflicts of Interest: All authors have completed the ICMJE uniform disclosure form (available at https://dx.doi. org/10.21037/atm-21-6356). The authors have no conflicts of interest to declare.

Ethical Statement: The authors are accountable for all aspects of the work in ensuring that questions related to the accuracy or integrity of any part of the work are appropriately investigated and resolved. Experiments were performed under a project license granted by Institutional Animal Care and Use Committee of Jilin University, in compliance with Institutional Animal Care and Use
Committee of Jilin University guidelines for the care and use of animals.

Open Access Statement: This is an Open Access article distributed in accordance with the Creative Commons Attribution-NonCommercial-NoDerivs 4.0 International License (CC BY-NC-ND 4.0), which permits the noncommercial replication and distribution of the article with the strict proviso that no changes or edits are made and the original work is properly cited (including links to both the formal publication through the relevant DOI and the license). See: https://creativecommons.org/licenses/by-nc-nd/4.0/.

\section{References}

1. Fassett RG, Coombes JS. Astaxanthin, oxidative stress, inflammation and cardiovascular disease. Future Cardiol 2009;5:333-42.

2. Zhang L, Wang H. Multiple Mechanisms of AntiCancer Effects Exerted by Astaxanthin. Mar Drugs 2015;13:4310-30.

3. Liu X, Luo Q, Rakariyatham K, et al. Antioxidation and anti-ageing activities of different stereoisomeric astaxanthin in vitro and in vivo. J Funct Foods 2016;25:50-61.

4. Park JS, Chyun JH, Kim YK, et al. Astaxanthin decreased oxidative stress and inflammation and enhanced immune response in humans. Nutr Metab (Lond) 2010;7:18.

5. Wu Q, Zhang XS, Wang HD, et al. Astaxanthin activates nuclear factor erythroid-related factor 2 and the antioxidant responsive element (Nrf2-ARE) pathway in the brain after subarachnoid hemorrhage in rats and attenuates early brain injury. Mar Drugs 2014;12:6125-41.

6. Zhang XS, Zhang X, Zhou ML, et al. Amelioration of oxidative stress and protection against early brain injury by astaxanthin after experimental subarachnoid hemorrhage. J Neurosurg 2014;121:42-54.

7. Kaptanoglu E, Palaoglu S, Surucu HS, et al. Ultrastructural scoring of graded acute spinal cord injury in the rat. J Neurosurg 2002;97:49-56.

8. Li GL, Brodin G, Farooque M, et al. Apoptosis and expression of Bcl-2 after compression trauma to rat spinal cord. J Neuropathol Exp Neurol 1996;55:280-9.

9. Yong C, Arnold PM, Zoubine MN, et al. Apoptosis in cellular compartments of rat spinal cord after severe contusion injury. J Neurotrauma 1998;15:459-72.

10. Liu XZ, Xu XM, Hu R, et al. Neuronal and glial apoptosis after traumatic spinal cord injury. J Neurosci 
Page 8 of 8

1997; 17:5395-406.

11. Crowe MJ, Bresnahan JC, Shuman SL, et al. Apoptosis and delayed degeneration after spinal cord injury in rats and monkeys. Nat Med 1997;3:73-6

12. Zhang XS, Zhang X, Wu Q, et al. Astaxanthin alleviates early brain injury following subarachnoid hemorrhage in rats: possible involvement of Akt/bad signaling. Mar Drugs 2014; 12:4291-310.

13. Wang CM, Cai XL, Wen QP. Astaxanthin reduces isoflurane-induced neuroapoptosis via the PI3K/Akt pathway. Mol Med Rep 2016;13:4073-8.

Cite this article as: Li S, Gao X, Zhang Q, Zhang X, Lin W, Ding W. Astaxanthin protects spinal cord tissues from apoptosis after spinal cord injury in rats. Ann Transl Med 2021;9(24):1796. doi: 10.21037/atm-21-6356

\section{Li et al. Astaxanthin protects the spinal cord tissues from injury}

14. Zhang M, Cui Z, Cui H, et al. Astaxanthin protects astrocytes against trauma-induced apoptosis through inhibition of NKCC1 expression via the NF- $\kappa \mathrm{B}$ signaling pathway. BMC Neurosci 2017;18:42.

15. Pan L, Zhou Y, Li XF, et al. Preventive treatment of astaxanthin provides neuroprotection through suppression of reactive oxygen species and activation of antioxidant defense pathway after stroke in rats. Brain Res Bull 2017;130:211-20.

(English Language Editor: L. Huleatt) 